\title{
Procalcitonin-Guided Antibiotic Discontinuation: An Antimicrobial Stewardship Initiative to Assist Providers
}

\author{
James J. Vaillant, MD, MSc, Tracey L. Mersfelder, PharmD, Rebecca S. Maynard, PharmD, \\ and Kevin Kavanaugh, MD
}

\section{ABSTRACT}

Background: Procalcitonin has emerged as an important marker of sepsis and lung infections of bacterial origin. The role of procalcitonin in guiding antibiotic stewardship in lower respiratory tract infections and sepsis has been extensively studied, and use of this biomarker has been shown to decrease antibiotic usage in clinical trials. We sought to evaluate the impact of a pharmacist-driven initiative regarding discontinuation of antibiotics utilizing procalcitonin levels at a community teaching hospital.

Methods: We retrospectively gathered baseline data on adult patients admitted to a community teaching hospital who were 18 years of age and older, under the care of an inpatient service, and had a single procalcitonin level $<0.25 \mathrm{mcg} / \mathrm{L}$ obtained during admission. We then prospectively identified an intervention group of similar patients using a web-based, real-time clinical surveillance system. When a low procalcitonin level was identified in the intervention group, the participating clinical pharmacists screened for antibiotic use and the indication(s), determined whether the antibiotic could be discontinued based on the low procalcitonin level and the absence of another indication for antibiotics, and, when appropriate, contacted the patient's health care provider via telephone to discuss possible antibiotic discontinuation. The total antibiotic treatment duration was compared between the baseline and intervention groups.

Results: A total of 172 patients were included in this study (86 in each group). The duration of antibiotic use was not significantly different between the baseline ( $3.14 \pm$ 4.04 days) and the intervention ( $3.34 \pm 2.8$ days) groups $(P=0.1083)$. Other patient demographics did not influence antibiotic duration.

Conclusion: Our study did not demonstrate a difference in total antibiotic treatment duration with the utilization of procalcitonin and an oral communication intervention made by a clinical pharmacist at a community-based teaching hospital. Outside of clinical trials, and in the absence of an algorithmic approach, procalcitonin has not consistently been shown to aid in the diagnosis and treatment of infectious diseases. It is important to have a comprehensive antimicrobial stewardship program to reduce antibiotic use and effectively use laboratory values.

Keywords: antibiotic use; bacterial infection; biomarkers; procalcitonin.
J

rocalcitonin is the precursor of the hormone calcitonin, which is normally produced in the parafollicular cells of the thyroid gland under physiological conditions. ${ }^{1}$ However, procalcitonin is also released in response to a proinflammatory stimulus, especially that of bacterial origin. 'The source of the procalcitonin surge seen during proinflammatory states is not the parafollicular cells of the thyroid, but rather the neuroendocrine cells of the lung and intestine. Stimulants of procalcitonin in these scenarios include bacterial endotoxin, tumor necrosis factor, and interleukin-6., ${ }^{1,2}$ Due to these observations, procalcitonin has emerged as an important marker of sepsis and lung infections of bacterial origin. ${ }^{3}$

The role of procalcitonin in guiding antibiotic stewardship in lower respiratory tract infections and sepsis has been extensively studied. ${ }^{4,5}$ Various randomized controlled trials

From Western Michigan University, Homer Stryker MD School of Medicine, Kalamazoo, MI (Dr. Vaillant and Dr. Kavanaugh), Ferris State University, Grand Rapids, MI (Dr. Mersfelder), and Bronson Methodist Hospital, Kalamazoo, MI (Dr. Maynard). 
Table 1. Demographic, Laboratory, and Vital Sign Data

\begin{tabular}{lcc}
\hline Variables & $\begin{array}{c}\text { Phase 1 } \\
\text { (Mean } \pm \text { SD) }\end{array}$ & $\begin{array}{c}\text { Phase 2 } \\
\text { (Mean } \pm \text { SD) }\end{array}$ \\
\hline Age, yr & $67 \pm 19$ & $69 \pm 16$ \\
\hline Duration of stay, days & $6.35 \pm 5.45$ & $6.6 \pm 4.5$ \\
\hline White blood cell count, $10^{\circ} / \mathrm{L}$ & $10.89 \pm 4.76$ & $9.98 \pm 4.39$ \\
\hline Temperature, maximum, ${ }^{\circ} \mathrm{F}$ & $98.7 \pm 0.98$ & $98.4 \pm 1.0$ \\
\hline
\end{tabular}

have shown that antibiotic stewardship guided by procalcitonin levels resulted in lower rates of antibiotic initiation and shorter duration of antibiotic use.4-6 Similar results were obtained in prospective studies evaluating its role in patients with chronic obstructive pulmonary disease and sepsis., ${ }^{7,8}$ Based on these data, protocol-driven procalcitonin-guided antibiotic stewardship appears beneficial.

Many of these studies employed rigorous protocols. Studies of procalcitonin use in a so-called real-world setting, in which the provider can order and use procalcitonin levels without the use of protocols, are limited. The objective of our study was to evaluate the impact of a pharmacist-driven initiative on discontinuing antibiotics, if indicated, utilizing single procalcitonin measurement results of $<0.25 \mathrm{mcg} / \mathrm{L}$ at a community teaching hospital.

\section{Methods}

Our study utilized a 2-phase approach. The first phase was a retrospective chart review to establish baseline data regarding adult inpatients with a low procalcitonin level; these patients were randomly selected over a 1-year period (2017). Patients were included if they were 18 years of age or older, under the care of an inpatient service, and had a single procalcitonin level $<0.25 \mathrm{mcg} / \mathrm{L}$ obtained during their admission. Patients admitted to the intensive care unit were excluded. In the second phase, we prospectively identified similar patients admitted between January and March 2018 using a web-based, real-time clinical surveillance system. When patients with low procalcitonin levels were identified, 2 participating clinical pharmacists screened for antibiotic use and indication. If it was determined that the antibiotic could be discontinued as a result of the low procalcitonin level and no additional indication for antibiotics was present, the pharmacist contacted the patient's health care provider via telephone to discuss possible antibiotic discontinuation. Data collected before and after the intervention included total antibiotic treatment duration, white blood cell count, maximum temperature, age, and procalcitonin level.

A sample size of 86 was calculated to provide an alpha of 0.05 and a power of 0.8. A nonparametric Wilcoxon 2-sample test was used to test for a difference in duration of antibiotic treatment between the baseline and intervention groups. A nonparametric test was used due to right-skewed data. All patients were included in the group analysis, regardless of antibiotic use, as the procalcitonin level may have been used in the decision to initiate antibiotics, and this is more representative of a real-world application of the test. This allowed for detection of a significant decrease of 2 days in antibiotic duration post intervention, with a $10 \%$ margin to compensate for potential missing data. Data from 86 patients obtained prior to the pharmacist intervention acted as a control comparison group. Statistical analysis was performed using SAS 9.4.

\section{Results}

A total of 172 patients were included in this study: 86 patients prior to the intervention, and 86 after implementation. Baseline demographics, laboratory values, vitals, and principal diagnoses for both groups are shown in Table 1 and Table 2. The most common indications for procalcitonin measurement were pneumonia (45.9\%), chronic obstructive pulmonary disease (15.7\%), and sepsis (14.5\%). The remaining diagnoses were encephalopathy, fever and leukocytosis, skin and soft tissue infection, urinary tract infection or pyelonephritis, bone and joint infection, meningitis, intra-abdominal infection, and asthma exacerbation.

Antibiotic therapy was initiated in $68 \%$ of the patients overall, 59\% in the baseline group and $76 \%$ in the intervention group. The duration of antibiotic use was not significantly different between the baseline $(3.14 \pm 4.04$ days $)$ and intervention (3.34 \pm 2.8 days) groups $(P=0.1083)$. Furthermore, antibiotic treatment duration did not vary significantly with patient age, white blood cell count, maximum temperature, or procalcitonin level in either group. Although there was no difference in total antibiotic treatment duration, a post-hoc analysis revealed a 
Table 2. Distribution of Diagnoses

\begin{tabular}{lccc}
\hline Principal Diagnosis & Phase 1, No. & Phase 2, No. & Total, No. (\%) \\
\hline Pneumonia & 38 & 41 & $79(45.9)$ \\
\hline Chronic obstructive pulmonary disease exacerbation & 6 & 21 & $27(15.7)$ \\
\hline Sepsis & 15 & 10 & $25(14.5)$ \\
\hline Encephalopathy & 4 & 6 & $10(5.8)$ \\
\hline Fever and leukocytosis & 8 & 2 & $10(5.8)$ \\
\hline Skin and soft tissue infection & 5 & 5 & $10(5.8)$ \\
\hline Urinary tract infection and pyelonephritis & 4 & 0 & $4(2.3)$ \\
\hline Bone and joint infection & 3 & 0 & $3(1.7)$ \\
\hline Meningitis & 2 & 0 & $2(1.2)$ \\
\hline Intra-abdominal infection & 1 & 1 & $1(0.6)$ \\
\hline Asthma exacerbation & 0 & $1(0.6)$
\end{tabular}

0.6-day decrease in the interval between the date of procalcitonin measurement and the stop date of antibiotics in the intervention group. The average time from admission to obtaining a procalcitonin level was 3 days in the baseline group and 2 days in the intervention group.

\section{Discussion}

Our study did not demonstrate a difference in total antibiotic treatment duration with procalcitonin measurement and an oral communication intervention made by a clinical pharmacist at a community teaching hospital with a well-established antimicrobial stewardship program. This may be due to several factors. First, the providers did not receive ongoing education regarding the appropriate use or interpretation of procalcitonin. The procalcitonin result in the electronic health record references the risk for progression to severe sepsis and/or septic shock, but does not indicate how to use procalcitonin as an aid in antibiotic decision-making. However, a recent study in patients with lower respiratory tract infections treated by providers who had been educated on the use of procalcitonin failed to find a reduction in total antibiotic use. ${ }^{9}$ Second, our study included hospital-wide use of procalcitonin, and was not limited to infections for which procalcitonin use has the strongest evidence (eg, upper respiratory tract infections, pneumonia, sepsis). Thus, providers may have been less likely to use protocolized guidelines. Last, we did not limit the data on antibiotic duration to patients with a procalci- tonin level obtained within a defined time frame from antibiotic initiation or time of admission, and some patients had procalcitonin levels measured several days into their hospital stay. While this is likely to have skewed the data in favor of longer antibiotic treatment courses, it also represents a more realistic way in which this laboratory test is being used. Our post-hoc finding of earlier discontinuation of antibiotics after procalcitonin measurement suggests that our intervention may have influenced the decision to discontinue antibiotics. Such an effect may be augmented if procalcitonin is measured earlier in a hospital admission.

Previous studies have also failed to show that the use of procalcitonin decreased duration of antibiotics. ${ }^{9,10}$ In the aforementioned study regarding real-world outcomes in patients with lower respiratory tract infections, antibiotic duration was not reduced, despite provider education. ${ }^{9}$ A large observational study that evaluated real-world outcomes in intensive care unit patients did not find decreased antibiotic use or improved outcomes with procalcitonin use..$^{10}$ With these large studies evaluating the 2 most common infectious diseases for which procalcitonin has previously been found to have clinical benefit, it is important for institutions to re-evaluate how procalcitonin is being utilized by providers. Furthermore, institutions should explore ways to optimize procalcitonin use and decrease unnecessary health care costs. Notably, the current community-acquired pneumonia guidelines recommend against routine use of procalcitonin. ${ }^{11}$ 
